\title{
Note: A robust low-cost high-sensitivity subangstrom bidirectional displacement sensor
}

\author{
P. R. Armitage ${ }^{1}$ and C. D. Wright ${ }^{2}$ \\ ${ }^{1}$ Theta Technologies Ltd., The Innovation Centre, University of Exeter, Exeter EX4 4RN, England \\ ${ }^{2}$ College of Engineering, Mathematics and Physical Sciences, University of Exeter, Exeter EX4 4QF, \\ England
}

(Received 16 June 2010; accepted 9 September 2010; published online 26 October 2010)

\begin{abstract}
The design of a simple, robust, low-cost, and bidirectional displacement sensor with subangstrom resolution is described. A small gold plate is attached to the object, whose displacement is to be measured, and a small gold tip is brought into contact with the plate, establishing a reference current between the two. Movements of the gold plate away from the tip lead to a change in the tip-plate current; the reference current is then re-established by moving the tip using a simple magnetic actuator. The resolution of the sensor has been calibrated using an interferometer and was shown to be as high as $0.3 \AA$ per system count. Potential applications of the sensor are numerous, including microbar air pressure measurement, motion and infrasound detection, thermal sensing, and gravitational field and relativistic gravity wave measurement. (c) 2010 American Institute of Physics. [doi:10.1063/1.3495964]
\end{abstract}

The measurement of displacement finds widespread application in the scientific, technological, medical, industrial, aerospace, and defense fields. Conventional, well-established approaches to detecting displacement include optical techniques (e.g., encoders and interferometers), electrical techniques (e.g., capacitive sensors and resistive sensors), electromagnetic techniques [e.g., the linear variable differential transformer (LVDT)], and strain-gauge approaches. ${ }^{1}$ A relative newcomer to the displacement sensing world, having its origins in the development of the scanning tunneling microscope (STM), is the tunneling sensor. A sensing technique based on tunneling offers potentially a huge sensitivity and the capability to measure extremely small displacements. Of course, the potential for using tunneling current as a displacement sensor has been known for over 20 years (see, for example, Ref. 2), but the design of such sensors has to date invariably used a cantilever approach "borrowed" from STM technology (see, for example, Refs. 3-7). While cantileverbased tunneling displacement sensors are undoubtedly highly sensitive and readily microfabricated, their use has so far been rather limited due in part to their relative lack of robustness, limited dynamic range, and relatively high cost. In this paper, we present an alternative concept for a tunneling based displacement sensor that bears little or no resemblance to conventional designs. First, our sensor is macroscopic in scale, rather than microscopic; it requires no microfabrication and can be readily constructed with elementary mechanical and electronic skills. Second, it is electromechanical in action with a direct digital (count) output and needs no complicated feedback control. Third, it is cantileverless, its active component being a simple cylindrical gold pin that is free to slide longitudinally (between guides). Finally, it is extremely robust and delivers subangstrom resolution together with a huge dynamic range (millimeters).

The basic sensor arrangement is shown in Fig. 1. Its principle of operation is extremely simple. The gold pin is free to slide in a rectangular tubelike mounting and can be attracted to a gold plate by an electromagnetic force controlled by a servocoil. The voltage between the pin and the plate is monitored, and if it rises above a predetermined (set) value, indicating an increase in the effective pin-plate resistance (a decrease in pin-plate current), a coil attached to the plate is energized by a short pulse (this will occur when the plate is moved to the right in Fig. 1). Energizing the coil attracts the small iron washer that is attached to the gold pin, bringing the point of the pin back into contact with the plate. This action occurs very quickly and the pin effectively tracks the motion of the plate by a series of short voltage pulses applied to the coil. As shown later (see Fig. 4), the pin tracks extremely small movements of the plate and, for a continuously moving plate, a tunneling current flows between the two. The pin itself is typically $<1 \mathrm{~mm}$ in diameter, with its end ground to a fine point, typically with a contact radius of around $100 \mu \mathrm{m}$ or so. The pin is supported and slides back and forth between two thin gold plates that have square holes cut in them; the round pin rests in the square holes, as shown in Fig. 2. The sliding surface of the pin has a very thin layer of mercury applied to improve the electrical contact between the slide supports and the pin. A standard soft iron washer (typically around $2 \mathrm{~mm}$ in diameter and $1 \mathrm{~mm}$ thick) is glued to the pin such that when the coil behind the plate is energized, the pin is pulled toward the plate. These energizing pulses are of very short duration, typically only a few nanoseconds long and are applied in rapid succession when the plate is moving freely. Thus, the sensor produces a direct digital count each time the pin is pulled back to the plate, yielding a linear displacement transducer that can potentially track long ranges of motion (millimeters) in nanoscale steps via simple electromagnetic actuation (between the pin and plate) and without the need for complicated feedback control. When first using the device, it is necessary to ensure that a "clean" pin-to-plate contact is produced. This is achieved by applying a relatively large voltage $(\sim 1.4 \mathrm{~V})$ between the pin and the plate. At this voltage, the electric field in the 


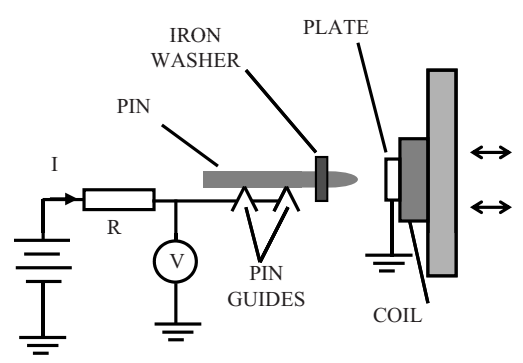

FIG. 1. Schematic of the sensor arrangement.

vicinity of the pin tip is very high, probably sufficient to cause an "explosive" electron emission that helps remove contaminants (e.g., water vapor) from the pin-plate contact region and to establish a reliable tunneling contact. After the tip cleaning process, the voltage is reduced to sensor operating levels and the pin-plate contact is reinstated. If the pin and the plate separate by a large amount during operation, then this electrode cleaning process must be repeated. Such a separation can occur if the device receives a large jolt and the pin-to-plate tracking is lost. The tip cleaning process can be repeated as often as is necessary, but in normal operation is infrequently required.

The system electronics, like the sensor itself, is exceedingly simple in its design and is shown in Fig. 3. A precision voltage source provides a user-selectable operating bias voltage in the range of $50-120 \mathrm{mV}$ or can be switched to provide a higher voltage $(\sim 1.4 \mathrm{~V})$ for tip cleaning as described above. The bias voltage is applied to the gold pin via a current limiting series resistor (here $1 \mathrm{k} \Omega$ ). The pin is also connected to the input of a very high impedance amplifier, the output of which feeds a regenerative comparator (Schmidt trigger). When the pin and plate are in contact, a current flows from the pin to the plate and the voltage at the pin drops to nearly zero. Should the plate move such that the pin is separated from it by a small distance, the current flow reduces, and the voltage on the pin rises and, via the amplifier, causes the comparator to switch from high to low. This switching event is passed to the coil driver circuit via a CMOS buffer, which, in turn, energizes the coil (attached to the pin assembly). With the coil energized, the pin is forced back on to the plate; the pin voltage then drops again to zero, the comparator resets, the voltage applied to the coil is turned off, and the system is ready for another plate movement. Each switch of the comparator also generates a count pulse for the system, with the number of counts acting as the

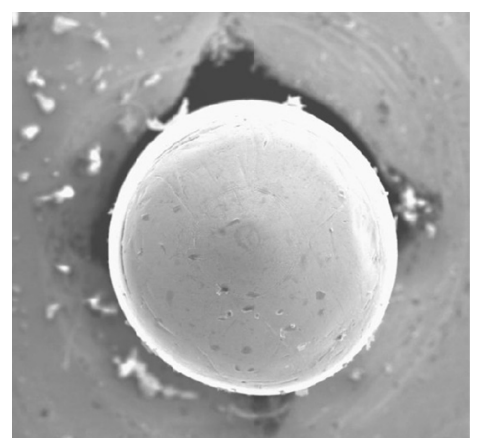

FIG. 2. SEM image of the pin (end-on view) and the gold supports in which it moves, showing one of the square cut-outs (image is $1.4 \mathrm{~mm}$ wide).
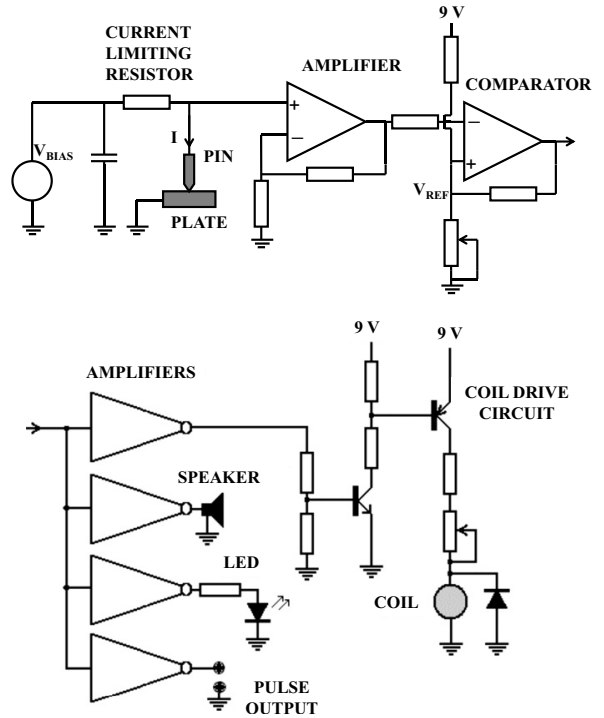

FIG. 3. Schematic of the electronic subsystem. The output from the comparator in the top figure feeds into the parallel (CMOS buffer) amplifiers at the bottom figure.

displacement measure. A visual (LED) output is also provided to indicate that the pin and the plate are tracking correctly, and an audio output of the coil pulses is included and has been found to be useful in setting up the sensor. Note that the process of pin separation from the plate to the coil being energized and the pin being restored on to the plate is very rapid, taking only a few nanoseconds. If the plate is in continuous motion, the process is repeated thousands of times a second, as the pin tracks the plate movement, each step separation producing a pulse. It has been found that the sensitivity (pulses per unit movement of the plate) of the sensor is able to be adjusted in a number of ways; the sensitivity is increased when the bias voltage supplied to the pin is increased (see Fig. 4). However, instability results if the bias voltage is increased too much and the repeatability worsens. The comparator switching voltage also controls the sensitivity, but again, instability will result if this is set too low, particularly if the system noise is relatively high (though the hysteresis in the Schmidt trigger provides protection against noise). As described, the system is sensitive to displacements only in one direction. It is, however, a simple matter to make a bidirectional sensor by the incorporation of a pin and a coil

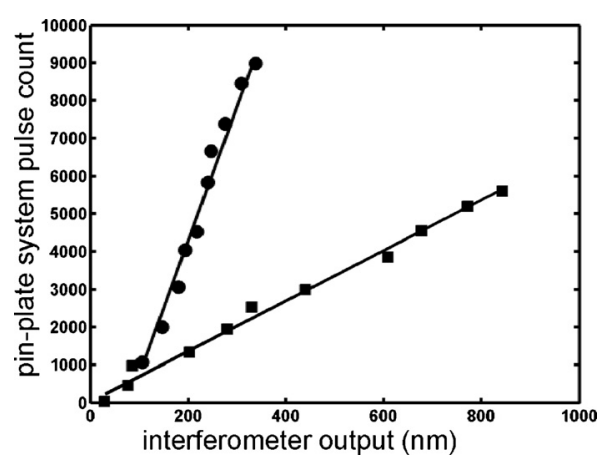

FIG. 4. Static tests comparing pin-plate output counts against interferometer measured displacement. Circles show the case for the $120 \mathrm{mV}$ bias and the squares for the $90 \mathrm{mV}$ bias. The resolution of the $120 \mathrm{mV}$ bias case corresponds to $0.3 \AA$ A count. 
arrangement on either side of the moving plate. This is shown in Fig. 5 (for application as an infrasound detector).

To determine the limits of performance of the sensor system, the pin-plate assembly was attached to a copper diaphragm and the diaphragm displaced mechanically over a small distance. An ultraprecision Jamin differential laser interferometer, ${ }^{8}$ designed by and housed in the National Physical Laboratories (Teddington, England), was used to calibrate the movement of the pin by comparing the output count from the sensor system to the interferometer measurement of displacement. For the interferometer measurement, the sensor pin was polished on its back face and a reference mirror attached a nonmoving mounting plate. The results from these static tests are shown in Fig. 4. For a bias of $120 \mathrm{mV}$, the sensor shows a remarkable resolution, for such a simple design, of $0.3 \AA$ Acount. This exceeds the resolution of the calibrating interferometer by around a factor of 10 and far exceeds that of most other standard displacement sensors (e.g., LVDTs, optical encoders, and strain gauges). It should also be noted that the dynamic range of the pin-plate sensor can be very large; in effect, since the pin is free to move in its guide plates, it is limited only by the length of the pin and the distance between guide plates. Again, this is in contrast to other conventional high-sensitivity displacement sensors, such as capacitive sensors, whose high resolution comes at the expense of a very limited dynamic range (and often a relatively high susceptibility to temperature changes too). The measurements of the repeatability of the pin-plate sensor were also made, at a lower sensitivity setting corresponding to $1.5 \AA /$ count, and showed a variance of \pm 0.4 count $/ \AA$ (and the repeatability was unaffected by tip cleaning). The resolution of the sensor is, we believe, primarily limited by the atomic thermal vibrations of the gold pin, which, at room temperature, correspond to a vibration amplitude of approximately $0.18 \AA .{ }^{9}$ Certainly, the electronics noise is not the limiting factor; the hysteresis for the Schmidt trigger in Fig. 3 was set to $20 \mathrm{mV}$, equivalent to an infinitesimal movement of the plate. The dynamic response of the sensor was also investigated briefly by attaching a swinging pendulum to the sensor plate to force it to oscillate sinusoidally and comparing the sensor output count to the interferometer displacement measurements. The sensor easily tracked oscillation amplitudes of $6 \mu \mathrm{m}$ at frequencies of $0.5 \mathrm{~Hz}$, equivalent to maximum linear plate velocities of $\sim 19 \mu \mathrm{m} \mathrm{s}^{-1}$. The limits of the dynamic performance are however much higher than this, with the main limiting parameters being the bandwidth of the electronics and the mechanical inertia of the pin. Using high bandwidth $(50 \mathrm{MHz})$, high slew rate $(10 \mathrm{~V} / \mu \mathrm{s})$ amplifiers should allow for the tracking of linear speeds of around $750 \mu \mathrm{m} \mathrm{s}^{-1}$ (equivalent to measuring a $\pm 100 \mathrm{~nm}$ movement at approximately $1.2 \mathrm{kHz}$ ).

There are many measurement applications for which the high sensitivity of the sensor would be most attractive. Examples include the measurement of microgradients of force, pressure, temperature, vibration, gravity, and electrical and magnetic fields. Thus, the pin-plate sensor may be suitable for applications as "mundane" as seismic surveying to those as exotic as gravity wave detection. An interesting application example, infrasound detection, is shown in Fig. 5. Here, a bidirectional version of the pin-plate sensor is configured to detect sound waves in the sub-40-Hz range, important in a
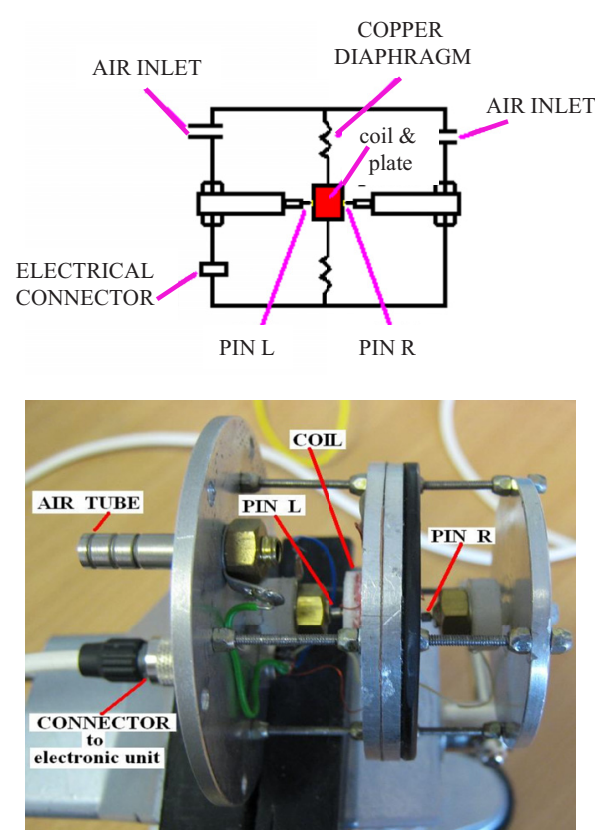

FIG. 5. (Color online) The sensor configured in bidirectional mode, in this case to be used as an infrasound detector. The top figure shows the schematic layout and the bottom figure is a photograph of the mechanical system.

number of defense applications as well as in the civil sphere (e.g., for avalanche warning). ${ }^{10}$ In the configuration shown in Fig. 5, the sensor can detect air pressure changes below $0.1 \mu$ bar $\left(10^{-2} \mathrm{~Pa}\right)$ at frequencies less than $40 \mathrm{~Hz}$. In all these potential applications, the ability of the pin-plate sensor to combine subangstrom level resolution with a wide dynamic range, the basic simplicity of the technology, its low energy consumption, its relative immunity to ambient conditions, and its potentially low manufacturing cost indicate that it should provide an attractive new approach to displacement sensing.

The authors are grateful to A-Metrics LLC of Charlotte, North Carolina, the patent holder that funded preparation of this paper; to B. Lubgins and L. Bekers for discussions on the original concept; to Dr. D. Robinson of Psi-tran Ltd. and Dr. K. Jackson and N. Cross of NPL, who organized and carried out the validation experiments; and to R. Perrett and $\mathrm{S}$. Coleman of Exeter University for their assistance with the device construction.

${ }^{1}$ J. Fraden, Handbook of Modern Sensors (Springer-Verlag, Berlin, 2004).

${ }^{2}$ M. F. Bocko, Rev. Sci. Instrum. 61, 3763 (1990).

${ }^{3}$ T. W. Kenny, S. B. Waltman, J. K. Reynolds, and W. J. Kaiser, Appl. Phys. Lett. 58, 100 (1991).

${ }^{4}$ T. W. Kenny, W. J. Kaiser, S. B. Waltman, and J. K. Reynolds, Appl. Phys. Lett. 59, 1820 (1991).

${ }^{5}$ D.DiLella, L. J. Whitman, R. J. Colton, T. W. Kenny, W. J. Kaiser, E. C. Vote, J. A. Podosek, and L. M. Miller, Sens. Actuators, A 86, 8 (2000).

${ }^{6}$ S. Sadewasser, G. Abadal, N. Barniol, S. Dohn, A. Boisen, L. Fonseca, and J. Estéve, Appl. Phys. Lett. 89, 173101 (2006).

${ }^{7}$ M. Dorrestijn, A. Bietsch, C. Gerber, and E. Myer, Nanotechnology 17, 2242 (2006).

${ }^{8}$ M. J. Downs and J. W. Nunn, Meas. Sci. Technol. 9, 1437 (1998).

${ }^{9}$ T. Comaschi, A. Balerna, and S. Mobilio, J. Phys.: Condens. Matter 21, 325404 (2009).

${ }^{10}$ A. J. Bedard and T. M. Georges, Phys. Today 53(3), 32 (2000). 